\title{
Toward weak Langmuir optical turbulence in a fiber cavity
}

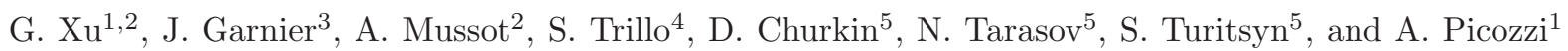 \\ ${ }^{1}$ Laboratoire Interdisciplinaire Carnot de Bourgogne, \\ CNRS, Université Bourgogne Franche-Comté, Dijon, France \\ ${ }^{2}$ Univ. Lille, CNRS, UMR 8523 - PhLAM - Physique des Lasers Atomes et Molécules, F-59000 Lille, France \\ ${ }^{3}$ Laboratoire de Probabilités et Modèles Aléatoires, \\ University Paris Diderot, 75205 Paris Cedex 13, France \\ ${ }^{4}$ Department of Engineering, University of Ferrara, Via Saragat 1, 44122 Ferrara, Italy \\ ${ }^{5}$ Aston Institute of Photonic Technologies, Aston University, Birmingham, UK
}

\begin{abstract}
We study theoretically and numerically the dynamics of a passive optical fiber ring cavity pumped by a highly incoherent wave - an incoherently injected fiber laser. The theoretical analysis reveals that the turbulent dynamics of the cavity is dominated by the Raman effect. The forced-dissipative nature of the fiber cavity is responsible for a large diversity of turbulent behaviors: Besides nonequilibrium statistical stationary states, we report the formation of a periodic pattern of spectral incoherent solitons, or the formation of different types of spectral singularities, e.g., dispersive shock waves and incoherent spectral collapse behaviors. We derive a mean-field kinetic equation that describes in detail the different turbulent regimes of the cavity and whose structure is formally analogous to the weak Langmuir turbulence kinetic equation in the presence of forcing and damping. A quantitative agreement is obtained between the simulations of the nonlinear Schrödinger equation with cavity boundary conditions and those of the mean-field kinetic equation and the corresponding singular integro-differential reduction, without using adjustable parameters. We discuss the possible realization of a fiber cavity experimental set-up in which the theoretical predictions can be observed and studied.
\end{abstract}

PACS numbers: 42.65.Tg,42.55.Wd,05.45.-a,42.81.Dp,42.65.Sf

\section{INTRODUCTION}

The propagation of partially coherent nonlinear optical waves is a subject of growing interest in different fields of investigations, such as, e.g., supercontinuum (SC) generation $[1,2]$, rogue waves [3-6], shock waves [7-9], nonlinear interferometry $[10,11]$, or incoherent wave propagation in homogeneous [12-16], discrete [17], multimode $[18,19]$ and single-mode fibers nearby the integrable limit [20-22]. From a different perspective, optical cavities and lasers offer an interesting experimental platform to study different regimes of optical turbulence [12, 23-37]. In particular, the phenomenon of condensation of photons has been recently demonstrated in optical microcavities [38], which raised important questions such as the relation between lasing and condensation [39]. Light condensation is expected to be associated to the natural thermalization of an optical wave in a nonlinear Kerr material. This irreversible process of thermalization is described in detail by the wave turbulence (WT) theory, which is inherently a nonequilibrium theory formally based on irreversible kinetic equations, as expressed by a $H$-theorem of entropy growth $[40,41]$. In particular, the WT formalism describes a process of wave condensation that can occur in an incoherently pumped passive optical cavity [42].

In this article we show that optical cavities can exhibit a turbulent regime of fundamental different nature than that discussed above. In this respect, we first note that the phenomenon of optical wave thermalization does not occur systematically, in the sense that it can be inhibited by different mechanisms, e.g., the presence of a nonlocal nonlinearity $[9,43]$ or the existence of additional invariants in generalized 1D nonlinear Schrödinger-like equations (NLSE) [1, 20, 44, 45]. Another mechanism responsible for a breakdown of optical wave thermalization is related to the causality condition underlying a noninstantaneous nonlinear response of the medium. A typical example is provided by the Raman effect in optical fibers. In this case the turbulent behavior of the random wave manifests itself in its spectral dynamics: The incoherent wave self-organizes into spectral incoherent solitons (SIS), i.e., incoherent solitons that cannot be identified in the spatio-temporal domain but solely in the spectral domain [46-51].

In this work we consider a passive optical fiber ring cavity pumped by an incoherent optical wave, whose coherence time, $t_{c}$, is much smaller than the round-trip time, $t_{c} \ll t_{R}[42,52,53]$. In this way, the optical beams from different cycles are mutually incoherent with one another, which makes the optical cavity non-resonant. As a consequence, the cavity is no longer modelled by the Lugiato-Lefever equation $[54,55]$. The analysis reveals that the system exhibits, as a general rule, a turbulent dynamics. The main difference with respect to the previous works $[42,53]$, is that we consider here a highly incoherent pump wave, in such a way that the Raman effect can no longer be neglected. The causality condition inherent to the Raman response function fundamentally changes the nature of the turbulent dynamics considered previously $[42,53]$. More specifically, the analysis reveals that the turbulent behavior is exclusively dominated by the Raman effect, whose kinetic description is found to be formally analogous to that used to describe weak Langmuir turbulence in plasmas $[56-58]$ (also see $[59,60]$ ). 
It is important to note that, from a broader perspective, Langmuir turbulence in the strongly nonlinear regime has been the subject of a huge number of theoretical and experimental studies [61, 62], in particular in the original context of hydrodynamics [62-65], or in controlled laboratory $[66,67]$ and space plasma experiments [68-70]. Furthermore, evidence of cavitating Langmuir turbulence has been recently shown to occur in natural Earth's aurora driven by solar wind [71]. To our knowledge however, besides preliminary experiments in [49], a clear experimental signature of weak Langmuir turbulence has not been reported in the context of nonlinear optics.

We discuss a formal analogy between the universal form of the kinetic equation describing the weakly nonlinear regime of Langmuir turbulence [58] and the kinetic equation derived here to describe an incoherently pumped passive optical fiber cavity. Indeed, we show that when the fiber ring cavity is pumped by a strongly incoherent wave, the system exhibits a turbulent dynamics which is described in detail by a mean-field kinetic equation (MF-KE) accounting for the incoherent pumping, the finesse of the cavity, the propagation losses and the delayed nonlinearity. The direct formal analogy between the weak Langmuir turbulence KE and the derived MF-KE mainly relies on two factors, (i) the presence of the optical incoherent pump excitation and the natural losses inherent to an optical cavity configuration, (ii) the analogy between molecular vibrations mediated by the optical Raman effect and the excitations of ion-sound waves mediated by the decay of plasma oscillations.

It is worth noting that, in spite of the formal analogy with weak Langmuir turbulence, in the optical context discussed here the nonlinear response function of the material is constrained by the causality condition, so that the (Raman-like) spectral gain function involved in the $M F-K E$ exhibits a long-range interaction in frequency space. In this way, the analysis of the MF-KE reveals the existence of nonequilibrium stationary turbulent states, whose balance among forcing, damping and nonlinearity can be either local or strongly nonlocal in frequency space. More generally, the forced-dissipative nature of the cavity system is responsible for a large diversity of non-stationary turbulent behaviors, such as the formation of a periodic pattern of continuous or discrete SISs, as well as the formation of spectral singularities, i.e., incoherent dispersive shock waves and incoherent spectral collapse singular behaviors. We stress the fact that the simulations of the stochastic NLSE describing the incoherent wave circulating in the cavity have been found in quantitative agreement with those of the MF-KE as well as the corresponding singular integro-differential reduction, without using adjustable parameters. In the first part of the article we discuss an ideal cavity configuration to provide a panoramic overview of different regimes of the turbulent cavity dynamics. In order to stimulate experiments, in the second part of the article we consider a specific realistic fiber ring cavity system. We finally note that this work can also shed new light on the peculiar role that plays the Raman effect on the generation of frequency combs in high-finesse optical micro-cavities [72-76], a subject of current intense investigations in relation with the important issue of the coherence properties of frequency combs [77-81].

\section{MODEL}

We study the temporal dynamics of a partially coherent wave that circulates in a passive optical fiber ring cavity pumped by a highly incoherent wave. The propagation of the optical wave in the fiber is known to be described by the standard generalized NLSE model [51]:

$$
\begin{array}{r}
-i \partial_{z} \psi(z, t)=\hat{d}\left(\partial_{t}\right) \psi(z, t)+\gamma\left(1+i \tau_{s} \partial_{t}\right) \psi(z, t) \\
\times \int_{-\infty}^{+\infty} \mathcal{R}\left(t^{\prime}\right)\left|\psi\left(z, t-t^{\prime}\right)\right|^{2} d t^{\prime}+i \alpha \psi(z, t),
\end{array}
$$

where $\hat{d}\left(\partial_{t}\right)=\sum_{j \geq 2} \frac{\beta_{j}}{j !}\left(i \partial_{t}\right)^{j}$ is the linear dispersion operator accounting for the lowest-order $\left(\beta_{2}\right)$, and higherorder dispersion effects, $\gamma$ refers to the nonlinear coefficient and $\mathcal{R}(t)=\left(1-f_{R}\right) \delta(t)+f_{R} R(t)$ to the usual response function accounting for the instantaneous Kerr effect and the non-instantaneous Raman response $R(t)$, which is constrained by the causality condition, $R(t)=0$ for $t<0$. The typical width of $R(t)$ denotes the nonlinear response time, $\tau_{R}$. Equation (1) also describes selfsteepening through the term proportional to $\tau_{s} \partial_{t}$, which accounts for the dispersion of the nonlinearity [51]. We remind that NLSE (1) conserves the 'number of photons', $N=\int|\tilde{\psi}(\omega, z)|^{2} /\left(1+\tau_{s} \omega\right) d \omega$, with $\tilde{\psi}(\omega, z)=$ $\frac{1}{2 \pi} \int \psi(t, z) e^{i \omega t} d t[82]$.

The cavity is pumped by a statistically stationary incoherent optical wave, with correlation function $\left\langle F(t) F^{*}\left(t^{\prime}\right)\right\rangle=C_{F}\left(t^{\prime}-t\right)$, such that $\left\langle\tilde{F}(\omega) \tilde{F}^{*}\left(\omega^{\prime}\right)\right\rangle=$ $\delta\left(\omega-\omega^{\prime}\right) S_{F}(\omega)$, with the power spectral density $S_{F}(\omega)=$ $\tilde{C}_{F}(\omega),\left(\tilde{F}(\omega)=\frac{1}{2 \pi} \int F(t) e^{i \omega t} d t\right)$. Note that, here and below throughout the paper, the brackets $\langle$. $\rangle$ denote an averaging over the realizations of the stochastic function $F(t)$. We denote by $t_{c}$ the coherence time (i.e., the width of $C_{F}$ ) and by $P_{F}=C_{F}(0)$ the average pump power. We denote by $F_{m}(t)$ the pump injected at $m$-th round-trip at time $t-m t_{R}$. We assume that the coherence time of the incoherent pump is much smaller than the round trip time, $t_{c} \ll t_{R}$ (the longitudinal coherence length is much smaller than the cavity length), so that $\left\langle\tilde{F}_{m}(\omega) \tilde{F}_{m^{\prime}}^{*}\left(\omega^{\prime}\right)\right\rangle=\delta\left(\omega-\omega^{\prime}\right) \delta_{m m^{\prime}}^{K} S_{F}(\omega), \delta_{m, m^{\prime}}^{K}$ denoting the Kronecker symbol. As discussed in previous works $[52,53]$, the passive cavity does not operate as a resonant 'phase-sensitive interferometer' [54, 55, 83-88], and the temporal modes of the cavity do not play any key role in the dynamics of the incoherent wave. The wave circulating in the cavity and the pump wave are thus mutually incoherent with each others, and the boundary 
conditions are not sensitive to the random relative phase among them:

$$
\psi_{m+1}(z=0, t)=\sqrt{\rho} \psi_{m}(z=L, t)+\sqrt{\theta} F_{m}(t),
$$

where $\psi_{m}(z, t)$ denotes the intracavity optical field after $m$ round trips $(0 \leq z \leq L)$, while $\rho$ and $\theta$ respectively refer to the reflection and transmission coefficients of the field intensity, $\rho+\theta=1$. Note that the boundary conditions (2) differ from those employed to derive the mean-field Lugiato-Lefever model $[54,55]$, where the coherent phase shift gives an additional parameter, namely the detuning, which critically affects the intracavity field dynamics. In contrast, in the incoherent case, only the finesse of the cavity is left to play an important role, $\mathcal{F}=2 \pi / \Gamma$, where $\Gamma=\theta+2 \alpha L$ denotes the effective amount of losses per round trip. The cavity finesse is related to the time required to fill an initially empty cavity, the so-called 'injection time' or average life-time that a photon spends in the cavity, $\tau_{p h}=t_{R} / \Gamma$. Once the cavity is filled, the average power of the intracavity optical wave reaches a stationary value, a feature that will become apparent through Eq.(15).

\section{MEAN-FIELD KINETIC EQUATION}

In this section we combine the KE that describes the evolution of the spectrum of the optical wave in the fiber $(0 \leq z \leq L)$ with the cavity boundary conditions (2) so as to derive the MF-KE describing the turbulent system. In the first step we ignore the cavity boundary conditions, i.e., we consider the propagation of the optical field through the fiber at the round trip $m\left(\psi_{m}(z, t)\right.$, $0 \leq z \leq L)$. A fundamental assumption of the wave turbulence theory used to derive the $\mathrm{KE}$ is that the random optical wave evolves in the weakly nonlinear regime, i.e., the highly incoherent regime in which linear dispersive effects dominate nonlinear effects $[12,40,41], t_{c} \ll \tau_{0}$, where $t_{c}$ is the coherence time of the incoherent wave, and $\tau_{0}=\sqrt{\left|\beta_{2}\right| L_{0} / 2}$ is the 'healing time', $L_{0}=1 /\left(\gamma P_{F}\right)$ being the nonlinear length. Notice that the 'healing time' denotes the time scale for which linear and nonlinear effects are of the same order, e.g., the typical time period of modulational instability [12]. In this weakly nonlinear regime, the statistics of the random wave results essentially Gaussian [12, 40, 41, 46, 47, 89], which allows one to achieve a closure of the hierarchy of moments equations. Starting from the NLSE (1), one obtains a KE governing the evolution of the averaged spectrum of the incoherent wave $\left\langle\tilde{\psi}_{m}(z, \omega+\Omega / 2) \tilde{\psi}_{m}^{*}(z, \omega-\Omega / 2)\right\rangle=n_{m}(z, \omega) \delta(\Omega)$ for $0 \leq z \leq L$ :

$$
\begin{array}{r}
\partial_{z} n_{m}(z, \omega)=\frac{\bar{\gamma}}{\pi} n_{m}(z, \omega) \int G\left(\omega, \omega^{\prime}\right) n_{m}\left(z, \omega^{\prime}\right) d \omega^{\prime} \\
-2 \alpha n_{m}(z, \omega),
\end{array}
$$

where $G\left(\omega, \omega^{\prime}\right)=\left(1+\tau_{s} \omega\right) g\left(\omega-\omega^{\prime}\right), \bar{\gamma}=f_{R} \gamma$, and $g(\omega)=\Im[\tilde{R}(\omega)]$ is the imaginary part of the Fourier transform of $R(t)$, which will be denoted as 'spectral gain function' in the following [50]. In the limit $\alpha=$ 0, Eq. (3) conserves the averaged number of photons, $\hat{N}_{m}=\int n_{m}(z, \omega) /\left(1+\tau_{s} \omega\right) d \omega$ over the round-trip. Note that the KE (3) accounts for nonlinear dispersive effects (self-steepening), but not for linear dispersion effects, although linear dispersion plays a key role in the establishment of the weakly nonlinear regime [12]. This means that the KE does not depend on the sign of the dispersion coefficient (normal or anomalous dispersion regime) and thus does not describe coherent soliton states. Note that the fact that the generation of a coherent soliton is quenched by the strong randomness of the incoherent wave in the weakly nonlinear regime $\left(t_{c} \ll \tau_{0}\right)$ has been already studied in different cases [90-92], in particular through supercontinuum generation $[12,93]$.

It is also important to remark that the instantaneous Kerr nonlinearity does not enter the KE (3). Indeed, the conservative four-wave interaction is known to achieve a closure of the hierarchy of moment equations at the second-order in the perturbation expansion procedure inherent to the WT theory [12]: To next order, the instantaneous Kerr nonlinearity coupled to higher-order dispersion leads to a collision term that describes, e.g., supercontinuum generation through optical wave thermalization [12]. We anticipate that the theory will be validated by the simulations, as revealed by the quantitative agreement obtained between NLSE and MF-KE simulations.

Let us now consider the boundary conditions of the passive cavity. Taking the Fourier transform of Eq.(2) and neglecting the correlations between the incoherent intracavity field and the incoherent pump field, we have

$$
n_{m+1}(z=0, \omega)=\rho n_{m}(z=L, \omega)+\theta S_{F}(\omega),
$$

where the averaged spectrum $S_{F}(\omega)$ of the pump field, such that $\left\langle\tilde{F}_{m}(\omega+\Omega / 2) \tilde{F}_{m}^{*}(\omega-\Omega / 2)\right\rangle=S_{F}(\omega) \delta(\Omega)$, is independent of the round trip $m$. Also note that the averaged pump power is given by $P_{F}=\int S_{F}(\omega) d \omega$.

In order to derive the MF-KE we need to assume that the averaged spectrum of the wave, $n_{m}(\omega, z)$, exhibits a slow variation within a single round trip. As defined above, the averaged spectrum denotes an average over the realizations of the injected stochastic pump wave. We note that, contrary to the usual mean-field approach underlying the Lugiato-Lefever equation $[54,55]$, here we do not assume that the field amplitude $\psi_{m}(t, z)$ exhibits a slow variation within a round trip - the individual fluctuations of the incoherent wave $\psi_{m}(t, z)$ exhibit rapid variations with a time correlation much smaller than the round-trip time, $t_{c} \ll t_{R}$ (or equivalently, the correlation length, $\lambda_{c}$, is much smaller than the cavity length, $\left.\lambda_{c} \ll L\right)$. It is important to note that, whenever one considers the high-finesse cavity regime, $\theta \ll 1, \alpha L \ll 1$, then the assumption that the averaged spectrum exhibits slow variations within a round-trip is automatically satisfied because we are considering the weakly nonlinear regime of interaction, $t_{c} \ll \tau_{0}$. The evolution of the kinetic Eq.(3) can then be averaged over a round trip by 
introducing the slow time derivative of the averaged spectrum, $\partial_{T} \tilde{n}(T, \omega)=\left[n_{m+1}(z=0, \omega)-n_{m}(z=0, \omega)\right] / t_{R}$, where $T=m t_{R}=m L / v_{g}, v_{g}$ being the group velocity of the optical field in the fiber. In this way we obtain the MF-KE

$$
\begin{aligned}
t_{R} \partial_{T} \tilde{n}(T, \omega)=\frac{\bar{\gamma} L}{\pi} \tilde{n}(T, \omega) & \int G\left(\omega, \omega^{\prime}\right) \tilde{n}\left(T, \omega^{\prime}\right) d \omega^{\prime} \\
& +\theta S_{F}(\omega)-\Gamma \tilde{n}(T, \omega),
\end{aligned}
$$

where $\Gamma=\theta+1-\exp (-2 \alpha L) \simeq \theta+2 \alpha L$ for $\alpha L \ll 1$. This MF-KE (5) provides a mean-field description $(\theta \ll 1$, $\alpha L \ll 1$ ) of the evolution of the averaged spectrum of the incoherent wave under the influence of the nonlinear interaction, the incoherent pumping, and both the cavity losses and the propagation losses. It describes the turbulent dynamics of the cavity with the following hierarchy of the relevant time scales, $t_{c} \ll \tau_{0} \ll t_{R}$. It is important to note that, because of the presence of the forcing and dissipative nature of the cavity, the MF-KE appears as the temporal counterpart of the weak Langmuir turbulence KE used to describe isothermal plasma in the presence of heavily damped ion-sound waves in the spatial domain $[57,58]$.

\section{STATIONARY STATES}

We start our study by considering the existence of turbulent regimes that are characterized by a stationary averaged spectrum, i.e., we look for the existence of stationary solutions to the MF-KE (5). We anticipate that the nonequilibrium stationary solution results from a balance (either local or nonlocal in frequency space), between forcing, damping and nonlinearity, so that their structure differs from the celebrated Kolmogorov-Zakharov spectra of turbulence, which are established in the inertial (conservative) regime of interaction $[40,41]$.

The stationary solutions extend in frequency space over a spectral bandwidth much larger than the typical bandwidth of the spectral gain function $g(\omega)$. The behavior of the tails of $g(\omega)$ are then expected to play a key role in the form of the stationary solution of the MF-KE. In this respect, we remind that because of the causality property of $R(t)$, the gain function $g(\omega)$ always decays algebraically at infinity, e.g., $\sim 1 / \omega^{3}$ for a damped harmonic oscillator, $\sim 1 / \omega$ for an exponential response. Such a long-range interaction in frequency space introduces singularities into the convolution operator of the MF-KE $(5), M_{\omega}(T)=\int g(\omega-u) \tilde{n}_{u}(T) d u$, which can be properly addressed by using the Hilbert operator, $\mathcal{H} f(\omega)=\pi^{-1} \mathcal{P} \int_{-\infty}^{+\infty} \frac{f(\omega-u)}{u} d u$, where $\mathcal{P}$ denotes the Cauchy principal value. It was shown in Ref.[7] that the convolution operator is dominated by the behavior of the response function $R(t)$ near by the origin $t=0$. More specifically, it was shown in the Supplement of Ref.[7] that the convolution operator can be written in the fol-
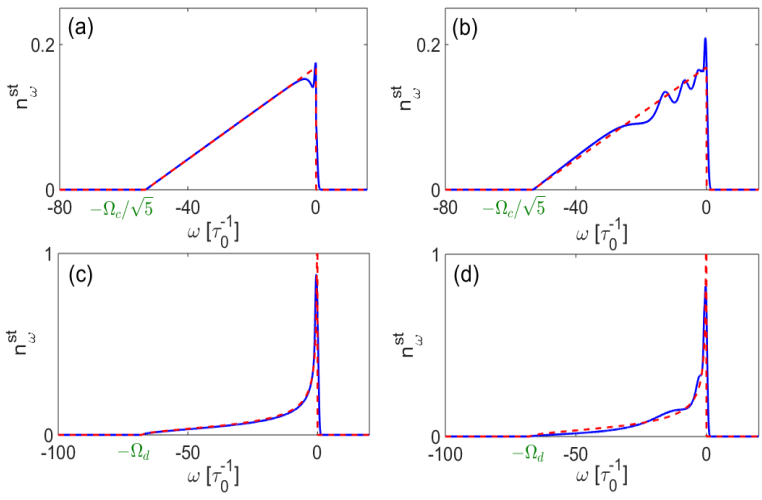

FIG. 1: Turbulent regimes characterized by a statistical stationary solution of the MF-KE (5). For a continuous (Ramanlike) response function, the stationary solution is given by (9) (red line, with $c=1 / \sqrt{5}$ ) (a-b); while for a discontinuous (exponential-like) discontinuous response function, the stationary solution is given by (14) (red line) (c-d). The stationary solutions are unstable, as revealed by the numerical simulations of the the MF-KE (5) (blue line), $z=50$ (a,c); $z=400(\mathrm{~b}, \mathrm{~d})$.

lowing form without approximations:

$$
\begin{aligned}
M_{\omega}(T)= & -\tau_{R}^{-1} \pi \bar{R}(0) \mathcal{H} \tilde{n}_{\omega}(T)+\tau_{R}^{-2} \pi \bar{R}^{(1)}(0) \partial_{\omega} \tilde{n}_{\omega}(T) \\
& +\frac{1}{2} \tau_{R}^{-3} \pi \bar{R}^{(2)}(0) \mathcal{H} \partial_{\omega}^{2} \tilde{n}_{\omega}(T) \\
+ & \tau_{R}^{-4} \int_{0}^{\infty}\left[\partial_{\omega}^{3} \tilde{n}_{\omega+\frac{u}{\tau_{R}}}+\partial_{\omega}^{3} \tilde{n}_{\omega-\frac{u}{\tau_{R}}}\right] G(u) d u
\end{aligned}
$$

where we have defined for $u>0: G(u)=-\frac{1}{2} \int_{u}^{\infty}\left(g\left(\frac{v}{\tau_{R}}\right)+\right.$ $\left.\frac{\bar{R}(0)}{v}-\frac{\bar{R}^{(2)}(0)}{v^{3}}\right)(v-u)^{2} d v$, and $\bar{R}(t)$ is a smooth function defined by $R(t)=\tau_{R}^{-1} \bar{R}\left(t / \tau_{R}\right) H(t), \bar{R}^{(n)}(0)$ denoting the $n$-th derivative at $t=0$ and $H(t)$ the Heaviside function. It is important to note that the expression (6) is exact and provides an expansion of the convolution operator $M_{\omega}(T)$ in powers of $1 / \tau_{R}$, without particular stringent assumptions on the specific form of the response function, $R(t)$.

In the following we will consider two different representative examples of response functions, either continuous or discontinuous at the origin, so as to illustrate qualitative different behaviors of the stationary turbulent solutions of the cavity. Note that, for simplicity, we will neglect in the following the impact of the self-steepening term, which simply introduces a multiplicative pre-factor $1+\tau_{s} \omega$ that plays a marginal role whenever the spectrum of the intracavity field evolves far from $-1 / \tau_{s}$.

\section{A. Continuous response function}

We illustrate the case of a continuous response function by considering the important example of the Raman-like 
damped harmonic response function [51]:

$$
R(t)=H(t) \frac{1+\eta^{2}}{\eta \tau_{R}} \sin \left(\frac{\eta t}{\tau_{R}}\right) \exp \left(-\frac{t}{\tau_{R}}\right) .
$$

This function being continuous at $t=0$, the first term in the expansion of the convolution operator (6) vanishes. On the other hand, the higher-order singular dispersive term (third term in (6)) can be neglected in the stationary turbulent regime considered in this section - while it plays a key role in the non-stationary regimes discussed below. Accordingly, the stationary solution of MF-KE (5), $n_{\omega}^{s t}$, that varies slowly compared to $1 / \tau_{R}$, is solution of the following reduced equation:

$$
\frac{\Omega_{c}}{N_{c}^{2}} n_{\omega}^{s t} \partial_{\omega} n_{\omega}^{s t}+\frac{\Omega_{c}}{2} \tilde{S}_{F}(\omega)-\frac{1}{N_{c}} n_{\omega}^{s t}=0,
$$

where we have introduced the characteristic frequency $\Omega_{c}=\sqrt{2\left(1+\eta^{2}\right) \theta \bar{\gamma} P_{F} L} /\left(\Gamma \tau_{R}\right)$, and characteristic spectral amplitude $N_{c}=\tau_{R} \sqrt{2 \theta P_{F} /\left(\left(1+\eta^{2}\right) \bar{\gamma} L\right)}$, while $\tilde{S}_{F}(\omega)=S_{F}(\omega) / P_{F}$. We are looking for a stationary solution $n_{\omega}^{s t}$ on a frequency interval that excludes the pump spectrum $\tilde{S}_{F}(\omega)$, i.e., $\omega \lesssim-\sigma_{F}$, where $\sigma_{F}$ denotes the typical spectral bandwidth of the pump wave. Then assuming $\Omega_{c} / \sigma_{F} \gg 1$, and $\tau_{R} \Omega_{c} \gg 1$ (which is a condition independent of $\tau_{R}$ ), the stationary solution reads:

$$
n_{\omega}^{s t}=N_{c}\left(\frac{\omega}{\Omega_{c}}+c\right)
$$

in the bulk spectrum away from the source $\tilde{S}_{F}(\omega)$, i.e., for $-c \Omega_{c} \leq \omega \lesssim-\sigma_{F}$, see Fig. 1a. Note that the constant of integration, $c$, can be chosen in such a way that $n_{\omega}^{s t}$ matches with the pump source nearby $\omega \sim 0$. This stationary solution results from a balance between the (Burgers) derivative operator and the losses, which are uniformly distributed in frequency space.

\section{B. Discontinuous response function}

Let us now consider the case of a discontinuous response function, which we illustrate with the familiar example of a purely exponential decay of the response:

$$
R(t)=H(t) \exp \left(-t / \tau_{R}\right) / \tau_{R}
$$

The discontinuity at $t=0$ completely changes the form of the stationary solution. According to the dominant singular term in the expansion of the convolution operator (6), we obtain the following reduced form of the stationary MF-KE:

$$
-\frac{1}{N_{d}^{2}} n_{\omega}^{s t} \mathcal{H} n_{\omega}^{s t}+\frac{\pi \Omega_{d}}{2} \tilde{S}_{F}(\omega)-\frac{1}{N_{d}} n_{\omega}^{s t}=0
$$

where we have introduced the characteristic frequency $\Omega_{d}=2 \theta \bar{\gamma} L P_{F} /\left(\pi \tau_{R} \Gamma^{2}\right)$ and spectral amplitude $N_{d}=$ $\tau_{R} \Gamma /(\bar{\gamma} L)$. We assume again that the pump spectrum
$S_{F}(\omega)$ is localized over a narrow frequency band at $\omega=0$ and look for a stationary solution on some interval $(-\Omega, 0)$, with $\Omega \gg \sigma_{F}$. We first note that, since $n_{\omega}^{s t}>0$ for $\omega \in(-\Omega, 0)$, the Hilbert transform $q_{\omega}=\mathcal{H} n_{\omega}^{s t}$ satisfies

$$
q_{\omega}=-N_{d} \text { for } \omega \in(-\Omega, 0) .
$$

Second, using the Poincaré-Bertrand identity $2 \mathcal{H}(f \mathcal{H} f)=(\mathcal{H} f)^{2}-f^{2}$, and $n_{\omega}^{s t}=-\mathcal{H} q_{\omega}$, we find that $q_{\omega}$ satisfies:

$$
\left(\mathcal{H} q_{\omega}\right)^{2}=q_{\omega}^{2}-\pi N_{d}^{2} \Omega_{d} \mathcal{H} \tilde{S}_{F}(\omega)+2 N_{d} q_{\omega} .
$$

Since $\mathcal{H} q_{\omega}=-n_{\omega}^{s t} \leq 0$, we have $n_{\omega}^{s t}=\left(q_{\omega}^{2}-\right.$ $\left.\pi N_{d}^{2} \Omega_{d} \mathcal{H} \tilde{S}_{F}(\omega)+2 N_{d} q_{\omega}\right)^{1 / 2}$. To pursue the analysis, we approximate the normalized pump spectrum by $\tilde{S}_{F}(\omega)=\delta(\omega)$, so that $\mathcal{H} \tilde{S}(\omega)=1 /(\pi \omega)$. Therefore, for $\omega \in(-\Omega, 0)$, we obtain $n_{\omega}^{s t}=N_{d}\left(-1-\Omega_{d} / \omega\right)^{1 / 2}$. This solution should be continuous away from the source, so this imposes $\Omega=\Omega_{d}$ and therefore the stationary solution reads:

$$
n_{\omega}^{s t}=N_{d} \sqrt{-1-\frac{\Omega_{d}}{\omega}} \text { for } \omega \in\left(-\Omega_{d}, 0\right) .
$$

We can check that this solution indeed satisfies $\int_{-\Omega_{d}}^{0} n_{\omega}^{s t} d \omega=\pi N_{d} \Omega_{d} / 2$, as expected by integrating (11) with respect to $\omega$. An illustrative plot of the stationary solution (14) is reported in Fig. 1b.

We remind that the stationary solution (14) of the MF$\mathrm{KE}$ results from a balance between the forcing, the dominant nonlinear singular term of the convolution operator, and the losses distributed uniformly in frequency space. Contrary to the previous case of continuous response function where such a balance is achieved 'locally' in frequency space as a result of the local property of the (Burgers) derivative operator, here, the Hilbert operator is inherently nonlocal, so that the balance among forcing, nonlinearity and losses results strongly delocalized in frequency space. As already commented, these nonequilibrium stationary solutions are of different nature than the Kolmogorov-Zakharov spectra of turbulence, which are solutions of the cubic nonlinear collision term of the kinetic equation in the inertial regime of interaction $[40,41]$. In particular, the stationary solutions discussed in this section are not characterized by a constant flux of particles (or energy) in frequency space.

In the next section we study the turbulent dynamics of the cavity by means of numerical simulations considering the natural experimental configuration where the cavity is initially empty and gets gradually filled by the injected incoherent pump wave. We note in this respect that numerical simulations performed by starting from the stationary solutions indicate that they are unstable, even in the absence of an additional perturbation (see Fig. 1). This is due to the fact that the stationary solutions refer to exact solutions of the stationary MF-KE in the 'bulk spectrum', i.e., far away from the frequency 
band that supports the pump source, $\omega \in\left[-\sigma_{F}, \sigma_{F}\right]$. Accordingly, the stationary solutions do not account for the specific form of the pump spectral shape, i.e., they do not match with the specific details of $S_{F}(\omega)$. This introduces a weak perturbation in the system, which is sufficient to destabilize the stationary solutions. This aspect has been also confirmed by remarking that stationary solutions featured by large values of $\Omega_{c}$ and $\Omega_{d}$ result more robust numerically, a feature which is consistent with the fact that such solutions are less sensitive to the details of the pump spectrum. We will see in the next section that, instead of the stationary solutions, the cavity develops a large diversity of non-stationary turbulent behaviors characterized by the emergence of spectral incoherent solitons or spectral singularities.

\section{HIGH-FINESSE CAVITY CONFIGURATION}

In this section we consider an 'ideal cavity configuration', in the sense that the finesse of the incoherently pumped cavity is deliberately enhanced in order to freely explore the large variety of turbulent regimes of the cavity. It is in this regime that the cavity deeply influences the turbulent dynamics, in the sense that the photon lifetime $\left(\tau_{p h}\right)$, is larger than all other relevant time scales it is larger than the time required for the formation of the pattern of SISs, or the time required for the development of spectral shock and collapse singularities. In the following, we compare in a systematic fashion the simulations of the incoherently pumped passive cavity based on the NLSE with those based on the MF-KE. Notice that all results of NLSE simulations reported below refer to a single simulation - no averaging over different simulations has been performed, while we implicitly perform an averaging over different realizations of the pump source since $F_{m}(t)$ is generated independently at each round-trip, see Sec. II. We study different turbulent regimes depending on the relative importance of the response time of the nonlinearity and the healing time, with $\tau_{R} \ll t_{R}$, $\tau_{0} \ll t_{R}$.

The NLSE simulations are performed by integrating Eq.(1) for the field $\psi_{m}(z, t)$ from $z=0$ to $z=L$, while $\psi_{m+1}(z=0, t)$ is computed by applying the boundary conditions given by the cavity map (2) at each round trip. The incoherent pump spectrum is assumed to be Gaussian-shaped, $S_{F}(\omega) \sim \exp \left[-\omega^{2} /\left(2 \sigma_{F}^{2}\right)\right]$. For convenience, we normalized the problem with respect to the pump power $P_{F}$, the nonlinear length $L_{0}$, the nonlinear time $T_{0}=L_{0} / v_{g}$, and the healing time $\tau_{0}$ [12]. The dimensionless variables are obtained through the transformations $z / L_{0} \rightarrow z ; \psi / \sqrt{P_{F}} \rightarrow \psi ; F / \sqrt{P_{F}} \rightarrow F ; \alpha L_{0} \rightarrow$ $\alpha ; L / L_{0} \rightarrow L ; t / \tau_{0} \rightarrow t$ and $T / T_{0} \rightarrow T=m L / L_{0}$, where we remind that $m$ denotes the number of round trips. Also note that a constant noise background has been added in the simulations. Such a spectral noise is important in order to sustain a steady incoherent soliton propagation [12], otherwise the SIS undergoes a slow
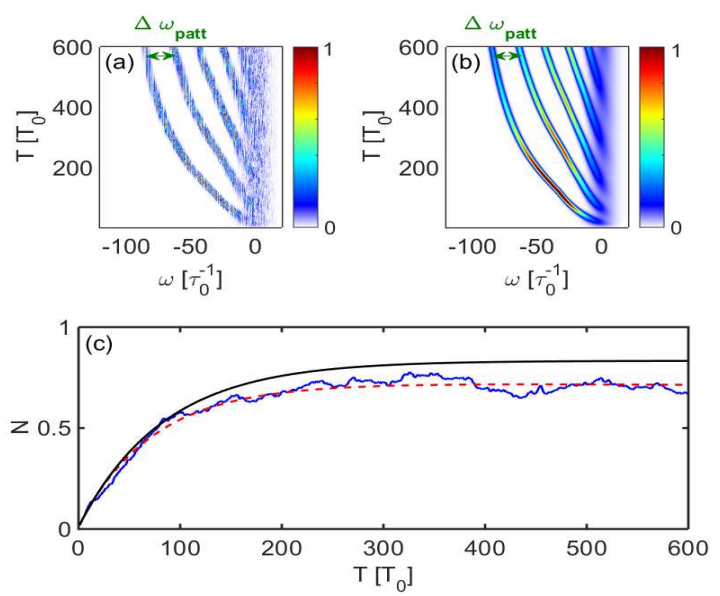

FIG. 2: Spectral pattern formation of SISs: Temporal evolution of the spectrum of the intracavity turbulent optical field obtained by solving the NLSE with boundary conditions Eqs.(1-2) (a), and the MF-KE (5) (b). (c) Corresponding evolutions of the intracavity power, $N(T)=\int \tilde{n}(T, \omega) d \omega$ : NLSE (1-2) (solid blue), MF-KE (5) (dashed red). Corresponding power corrected by the self-steepening factor, $\hat{N}(T)=$ $\int \hat{n}(T, \omega) d \omega$ (solid black), which relaxes toward a stationary state as predicted by Eq.(15), $\hat{N}^{s t}=\frac{\theta}{\Gamma} \hat{P}_{F} \simeq 0.83$. Parameters: $L=5 L_{0}$ is the total length of the cavity, $\eta=1, \tau_{R}=0.2$ in the response function, pump spectral bandwidth $\sigma_{F}=2 \pi$, losses $\alpha L=10^{-3}, f_{R}=0.18, \tau_{s}=1 / 280, \operatorname{sign}\left(\beta_{2}\right)>0$. Note that the typical SIS spectral width is given by the bandwidth of the spectral gain function, $\Delta \omega_{g}$, so that the spectral interval among adjacent SISs in (a-b) is much larger than $\Delta \omega_{g}$, i.e., $\Delta \omega_{\text {patt }} \gg \Delta \omega_{g}$.

adiabatic reshaping so as to adapt its shape to the local value of the noise background. This noise background can also simulate the presence of a quantum noise background. The amount of noise background is defined from the ratio, say $\mu=P_{n} / P_{F}$, between the background noise power $\left(P_{n}\right)$ and the average power of the injected pump wave $\left(P_{F}\right)$. In the following, otherwise stated, we have considered the typical of $\mu \simeq 5.1 \times 10^{-7}$, which is of the order of the quantum noise background level.

\section{A. Formation of a spectral pattern of SISs}

We start the simulations by investigating the possibility to generate SISs in the incoherently pumped passive cavity. For this purpose, we consider a spectral bandwidth of the incoherent pump, $\sigma_{F}$, of the same order as the corresponding bandwidth of the spectral gain function, $\sigma_{F} \sim \Delta \omega_{g} \sim 1 / \tau_{R}$. The response function implemented in the simulations refers to the Raman-like damped harmonic oscillator given in Eq.(7). In the following we will always consider the natural experimental configuration in which the cavity is initially empty, while the incoherent pump progressively fills the cavity in time. 
The cavity is characterized by a length $L=5 L_{0}$, while the amount of losses $\left(\alpha L=10^{-3}\right)$ has been chosen in such a way to enhance the cavity finesse, so that the intracavity power reached in the stationary regime is of the same order than the injected pump power. Note that concrete experimental values of the normalized parameters used in the simulations reported in this section are discussed in detail in Sec. VI. For instance, the simulations reported in Figs. 2-3 typically correspond to the experimental parameters given through the first line of Fig. 7 in Sec. VI.

The simulations reveal that the intracavity field exhibits a turbulent dynamics characterized by statistically stationary fluctuations. The cavity is filled according to the solution of MF-KE for $\hat{N}(T)=\int \tilde{n}(T, \omega) /\left(1+\tau_{s} \omega\right) d \omega$ : The 'number of photons' evolves according to

$$
\hat{N}(T)=\frac{\theta}{\Gamma}\left[1-\exp \left(-\frac{\Gamma T}{t_{R}}\right)\right] \int \frac{S_{F}(\omega)}{1+\tau_{s} \omega} d \omega .
$$

This means that it relaxes exponentially, with the characteristic photon life-time $\tau_{p h}=t_{R} / \Gamma$, toward the stationary state, $\hat{N}^{s t}=\theta \hat{P}_{F} / \Gamma$, with $\hat{P}_{F}=\int \frac{S_{F}(\omega)}{1+\tau_{s} \omega} d \omega$. This prediction is confirmed by the numerical simulations, as illustrated in Fig. 2c.

The main result revealed by the simulations is the formation of a spectral pattern of almost regularly spaced SISs. This is remarkably illustrated in Fig. 2a-b, which reports the temporal evolution of the spectrum of the turbulent field starting from an empty cavity. In a first stage, due to the high cavity finesse, a small amount of the incoherent pump enters the cavity, so that the system evolves in the linear regime. Once the intracavity pump intensity becomes large enough, the nonlinear regime leads to the generation of a SIS, which propagates in the spectral domain toward the low-frequency components. As a consequence, the frequency interval of incoherent pump excitation, $\omega \in\left[-\sigma_{F}, \sigma_{F}\right]$, results to be cleared out by the emission of the SIS. In this way, the process can start again: The intracavity pump is regenerated and once its intensity becomes large enough, a second SIS is emitted and is red-shifted away from the pump frequency band, and so on the process is repeated, thus leading to the formation of a regular spectral pattern of SISs, as evidenced in Fig. 2a-b. The resulting spectral pattern of SISs then spans several orders of the resonant Raman frequency shift. The cavity losses combined with the self-steepening effect, lead to a slow down of the most spectrally shifted SISs. Actually, the spectral bandwidth of the pattern of SISs is essentially limited by the finesse of the cavity, $\mathcal{F}=2 \pi / \Gamma$.

One may wonder whether the spectral pattern of SIS reported here can be explained by a mechanism of cascaded Raman scattering, a well-known process that has been widely studied in both single pass propagation $[94,95]$, or in cavity systems, e.g., in cw fiber lasers [96], in mode-locked fiber lasers [97], or in passive microresonators [98, 99]. Cascaded Raman scattering refers to the generation of several Stokes orders, the 1st order subsequently leading to the generation of the 2nd order, which in turn generates the 3rd one, and so on several orders are generated in cascade. In this way the different Stokes bands have fixed frequencies in the spectrum, and adjacent bands are separated one from each other by the Raman resonant frequency $\left(\Delta \omega_{g} \simeq 13.2 \mathrm{THz}\right.$ in conventional silica fibers). These properties contrast with the spectral pattern of SIS discussed here since, (i) each individual band is itself continuously frequency shifted during the temporal evolution, (ii) the frequency separation among adjacent bands is much larger than the Raman resonant frequency, $\Delta \omega_{\text {patt }} \gg \Delta \omega_{g}$ (see Fig. 2a). These fundamental differences with cascaded Raman scattering are not surprising since, as explained here above, in our system the mechanism underlying the formation of the spectral pattern of incoherent frequency bands is based on the emission of SISs from the injected incoherent pump. In other words the different bands in our spectral pattern do not correspond to different Stokes orders, but to the successive emission of different SISs from the injected incoherent pump. As a consequence, it is difficult to assess even qualitatively the frequency separation among adjacent SISs, since such a shift is related to the processes of SIS emission and pump regeneration in the cavity (which obviously depends on the cavity length and finesse).

Also note that the frequency shift of a SIS is very different from the well-known soliton self-frequency shift due to intra-pulse Raman scattering [51], since this effect is fundamentally related to the propagation of a coherent soliton pulse. In contrast, there is no pulse confinement in the temporal domain here, since we deal with an incoherent wave characterized by fluctuations that are statistically stationary in time.

A continuous SIS is known to become unstable under certain conditions, and can thus relax during the propagation toward a discrete SIS [49]. Indeed, depending on different key parameters, such as e.g., the spectral bandwidth of the pump, the amount of noise background, or the structure of the response function $g(\omega)$, the cavity system can self-organize into a spectral pattern of discrete SISs, as illustrated in Fig. 3. The discrete SIS is characterized by three incoherent spectral bands equally spaced by the Raman frequency: a new Raman band grows up by absorbing the previously generated spectral band, thus leading to a 'discrete propagation' of the discrete SIS in frequency space. As for continuous SISs, the cavity finesse also delimits the spectral bandwidth of the generated spectral pattern of discrete SIS.

We also verified by NLSE simulations that perturbative higher-order dispersion effects do not affect the spectral dynamics of the incoherent wave, in agreement with the theory. Notice the remarkable agreement between numerical simulations of the NLSE (1) with boundary conditions (2), and the simulations of the MF-KE (5), without using adjustable parameters. 

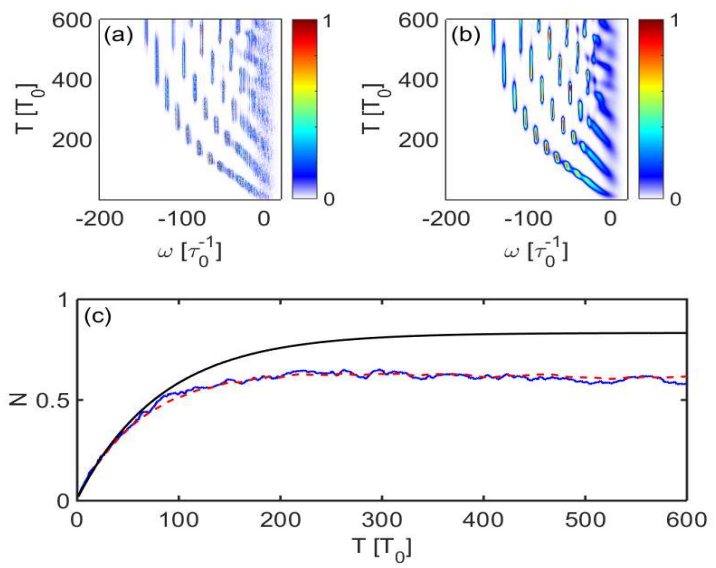

FIG. 3: Spectral pattern formation of SISs: Temporal evolution of the spectrum of the intracavity turbulent optical field obtained by solving the NLSE (1-2) (a), and the MF-KE (5) (b). (c) Corresponding evolutions of the intracavity power, $N(T)=\int \tilde{n}(T, \omega) d \omega:$ NLSE (1-2) (solid blue), MF-KE (5) (dashed red). Corresponding power corrected by the selfsteepening factor, $\hat{N}(T)=\int \hat{n}(T, \omega) d \omega$ (solid black), which relaxes toward a stationary state as predicted by Eq.(15), $\hat{N}^{s t}=\frac{\theta}{\Gamma} \hat{P}_{F}=0.83$. Parameters are the same as in Fig. 2, except that $\eta=2.6$, which entails a larger velocity of the soliton in frequency space (note that the frequency windows are different for Figs. 2 and 3).

\section{B. Incoherent spectral singularities}

In the previous section we discussed the turbulent regime of the cavity when the spectral bandwidth of the incoherent pump is of the same order as the bandwidth of the spectral gain function, $g(\omega)$. Let us now consider the regime in which the pump spectral bandwidth is increased in a significant way, $\sigma_{F} \gg \Delta \omega_{g} \sim 1 / \tau_{R}$. The cavity then enters a qualitatively different turbulent regime. As already discussed above in Sec. IV through the analysis of the stationary solutions, in this 'long-range regime' the behavior of the tails of the gain spectrum $g(\omega)$ plays an important role in the spectral dynamics. We anticipate that the cavity will be shown to exhibit a turbulent dynamics featured by the formation of spectral singularites, whose structures are described in detail by the expansion of the convolution operator $M_{\omega}$ given in Eq.(6). Proceeding as in Sec. IV, we will consider two different examples of continuous and discontinuous response functions to illustrate different forms of spectral singularities. We will also neglect the impact of the self-steepening term, since the spectral dynamics of the cavity will be shown to evolve far away from $-1 / \tau_{s}$, as will be confirmed by the numerical simulations reported in Figs. 4-5.
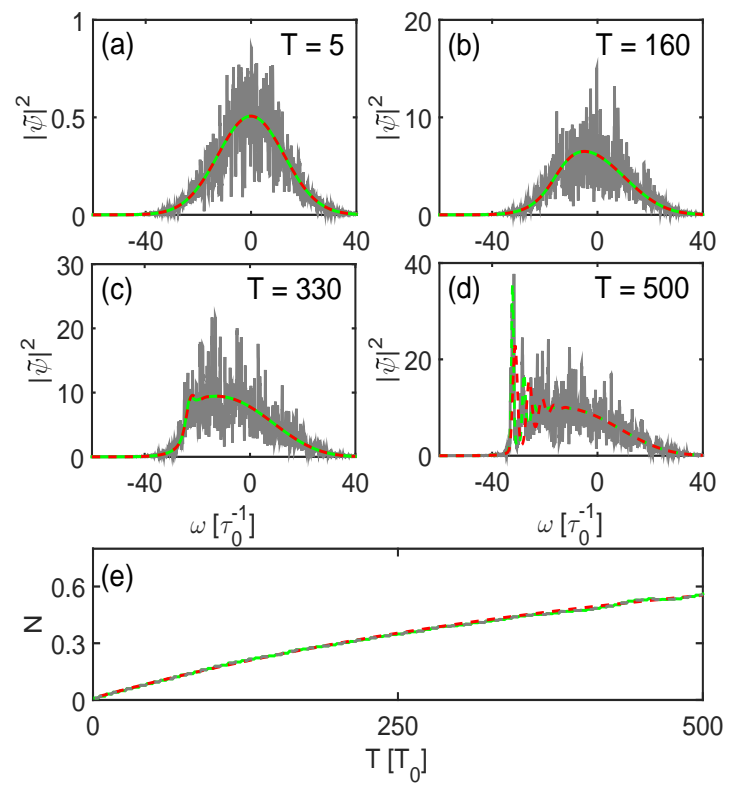

FIG. 4: Development of dispersive spectral incoherent shock waves (with continuous response function): (a-d) Temporal evolution of the spectrum of the intracavity turbulent optical field obtained by solving the NLSE (1-2) (gray line), the MF$\mathrm{KE}(5)$ (dashed-red line), the singular integro-differential MFKE (16) (dashed-green line). (e) Corresponding evolutions of the intracavity power, $N(T)=\int \tilde{n}(T, \omega) d \omega$ : The cavity develops the shock well before reaching the stationary steady state (15), $\tau_{\text {shock }} \ll \tau_{p h}$. Parameters: $L=20 L_{0}$ is the total length of the cavity, $\eta=1, \tau_{R}=2 \tau_{0}$ in the response function, pump spectral bandwidth $\sigma_{F}=4 \pi$, losses $\alpha L=10^{-3}, f_{R}=$ 0.18 .

\section{Continuous response function: Spectral shock wave}

We first consider the Raman-like response function to illustrate the example of the continuous response function. On the basis of the expansion of the convolution operator discussed through Eq.(6), the MF-KE for the averaged spectral dynamics $\tilde{n}_{\omega}(T)$ takes the form

$$
\begin{array}{r}
t_{R} \partial_{T} \tilde{n}_{\omega}=\frac{\bar{\gamma} L\left(1+\eta^{2}\right)}{\tau_{R}^{2}}\left(\tilde{n}_{\omega} \partial_{\omega} \tilde{n}_{\omega}-\frac{1}{\tau_{R}} \tilde{n}_{\omega} \mathcal{H} \partial_{\omega}^{2} \tilde{n}_{\omega}\right) \\
+\theta S_{F}(\omega)-\Gamma \tilde{n}_{\omega} .
\end{array}
$$

The leading-order Burgers term in (16) is responsible for the development of a gradient catastrophe, which is subsequently regularized by the nonlinear dispersive term involving the Hilbert operator [7]. This prediction is confirmed by numerical simulations of the NLSE (1) with boundary conditions (2), which have been found in quantitative agreement with the MF-KE (5) and the reduced singular integro-differential MF-KE (16), as shown in Fig. 4. As discussed in detail in [7], the dispersive shock wave reported here develops in the spectral evolution of the incoherent wave. It is thus of fundamental different nature than the conventional dispersive shocks that develop either in the spatial or the temporal domain 


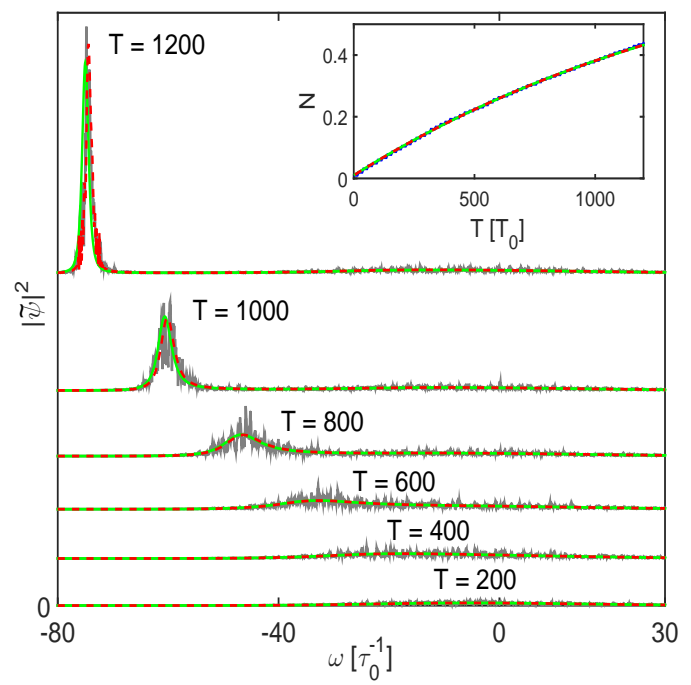

FIG. 5: Development of spectral collapse singularity (with discontinuous response function): Temporal evolution of the spectrum of the intracavity turbulent optical field obtained by solving the NLSE (1-2) (gray line), the MF-KE (5) (dashed-red line), the singular integro-differential MF-KE (17) (dashed-green line). The inset shows the corresponding evolutions of the intracavity power, $N(T)=\int \tilde{n}(T, \omega) d \omega$ : The cavity develops the collapse before it reaches the statistical stationary regime, $\tau_{\text {coll }} \ll \tau_{p h}$ Parameters: $L=20 L_{0}$ is the total length of the cavity, pump spectral bandwidth $\sigma_{F}=6 \pi, \tau_{R}=3 \tau_{0}, \eta=1$, losses $\alpha L=10^{-3}, f_{R}=0.18$.

from coherent disturbances, which have been experimentally observed in ion-acoustic waves [100], water surface gravity waves [101], and fiber optics [102], and have recently regained great interest in optics [103-112]. Coherent dispersive shocks and their stationary analogues have shown to play a role also in passive cavity configurations [113-115], where one can envisage that they can impact the generation of combs in the normal dispersion regime $[116,117]$.

Note that, in the incoherent case examined here, the incoherent shock singularity develops in the cavity well before that it reaches the statistically stationary steady state. In other words the characteristic shock time scale is much smaller than the photon life-time, $\tau_{\text {shock }} \ll \tau_{p h}$, see Fig. 4. This is interesting when one reminds the fact that the rapidly oscillating dispersive shock wave structure is known to regularize the shock singularity in a conservative (Hamiltonian) system. Here, the dispersive shock wave develops far from the statistical stationary regime, i.e., in the non-conservative regime where the injected pump is still filling the cavity, $\partial_{T} N(T)>0$.

\section{Discontinuous response function: Spectral collapse}

To illustrate the example of a discontinuous response function, we consider a purely exponential decay given in (10). The discontinuity at $t=0$ completely changes the dynamics [7], which is now dominated by a nonlinear singular term:

$$
\begin{aligned}
t_{R} \partial_{T} \tilde{n}_{\omega}= & \frac{\bar{\gamma} L}{\tau_{R}}\left(-\tilde{n}_{\omega} \mathcal{H} \tilde{n}_{\omega}-\frac{1}{\tau_{R}} \tilde{n}_{\omega} \partial_{\omega} \tilde{n}_{\omega}\right. \\
& \left.+\frac{1}{2 \tau_{R}^{2}} \tilde{n}_{\omega} \mathcal{H} \partial_{\omega}^{2} \tilde{n}_{\omega}\right)+\theta S_{F}(\omega)-\Gamma \tilde{n}_{\omega} .
\end{aligned}
$$

The impact of the leading-order term in (17) was discussed in detail in Ref.[7], in relation with an analytical solution originally derived in [118]. It was shown that the spectrum exhibits a collapse-like behavior, while the spectral peak is shifted toward the low-frequency components $(\omega<0)$ with a constant velocity. This general behavior is confirmed by the numerical simulations of the turbulent cavity, although the cavity operates far from the statistical stationary regime in the presence of a significant forcing, i.e., the collapse time is much smaller than the photon life-time, $\tau_{\text {coll }} \ll \tau_{p h}$, see the inset of Fig. 5 with $\partial_{T} N(T)>0$. Note again the quantitative agreement between NLSE (1), MF-KE (5) and the singular integro-differential MF-KE (17), without adjustable parameters.

\section{MODERATE FINESSE CAVITY CONFIGURATION}

\section{A. Numerical results}

Let us now discuss a more realistic fiber ring cavity configuration characterized by a moderate finesse. A typical experimental set-up of the fiber ring cavity is reported in Fig. 6. To be concrete, we considered here a cavity of length $L=25 \mathrm{~m}$, with fiber nonlinear coefficient $\gamma=2 \mathrm{~W}^{-1} \mathrm{~km}^{-1}$, which operates in the anomalous dispersion regime $\beta_{2}=-10^{-26} \mathrm{~s}^{2} / \mathrm{m}$ at the carrier pump wavelength $\lambda_{p}=1550 \mathrm{~nm}(193.5 \mathrm{THz})$. We remind here that in the strongly incoherent (i.e., weakly nonlinear) regime the sign of second-order dispersion does not affect the turbulent dynamics, as discussed above through the kinetic theory and the MF-KE. However, we will see that the weakly nonlinear regime is not guaranteed as the spectral bandwidth of the injected incoherent pump is decreased, which can be responsible for the generation of coherent soliton states. In order to increase the finesse of the cavity, we considered in the simulations a coupler of efficiency 0.99 , weak fiber losses $0.2 \mathrm{~dB} / \mathrm{km}$, as well as a strong (average) pump power of $P_{F}=100 \mathrm{~W}$ that enables a reduction of the length of the cavity. Note that such a high power source can be implemented in practice by considering a cavity synchronously pumped by incoherent long pulses. With these parameters we have a typical cavity finesse of $\mathcal{F} \simeq 500$. Simulations of the NLSE include both the instantaneous and the delayed contributions to the nonlinear response function, with the usual Raman contribution $f_{R}=0.18$ and response function $R(t)=\frac{1+\eta^{2}}{\eta \tau_{R}} \sin \left(\eta t / \tau_{R}\right) \exp \left(-t / \tau_{R}\right)$, with $\eta=2.6$ and 


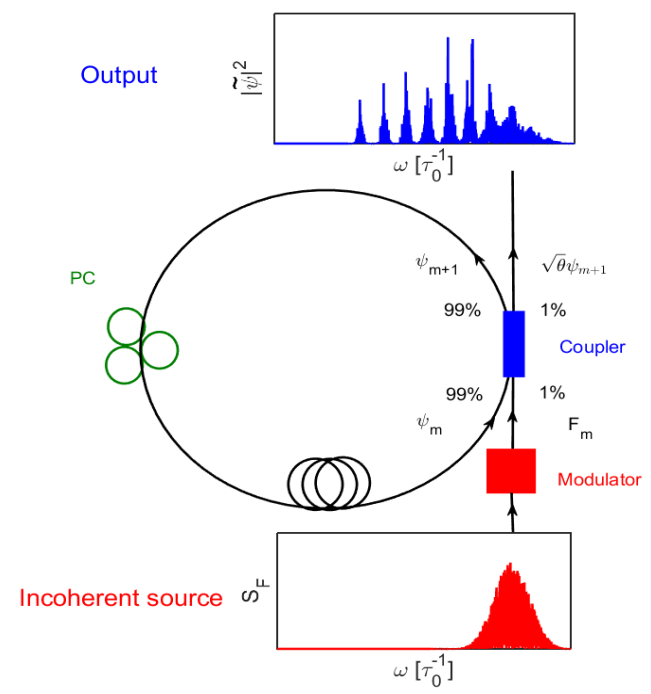

FIG. 6: Scheme of the fiber ring cavity experiment. The incoherent source can be modulated in time so as to synchronously pump the cavity with high power incoherent long pulses. The polarization controller can be used to get rid of fiber birefringence. A high-efficiency coupler is inserted in the cavity to increase the finesse. Numerical simulations of the NLSE reveal that the cavity generates a spectral pattern of SISs.

$\tau_{R}=32 \mathrm{fs}$. Note that this expression refers to the usual expression of the Raman response function in optical fibers with $\tau_{R}=32 \mathrm{fs}$ and $\eta=2.6\left(\tau_{1}=\tau_{R} / \eta=12.2 \mathrm{fs}\right)$ [51].

We report in Fig. 7 the results of the numerical simulations of the NLSE (1) with boundary conditions (2). Because of the large spectral bandwidth of Raman gain in optical fibers (i.e., the small response time $\tau_{R}$ ) the simulations reveal that the cavity usually operates in the regime characterized by the formation of a spectral pattern of SISs. The dynamics of the cavity is reported for different values of the spectral bandwidth of the incoherent pump, as illustrated by Fig. 7, where the pump bandwidth is varied from $6.2 \mathrm{THz}$ to $3.1 \mathrm{THz}$. More specifically, for small spectral bandwidths, the generated SISs exhibit a discrete behavior. Conversely, for larger spectral bandwidths, the cavity initially tends to generate a pattern of continuous SISs. However, each of the continuous SIS subsequently decays toward a stable discrete SIS, in analogy with a previous study of SIS emerging from a supercontinuum spectrum [49].

It is also interesting to note that, by decreasing the pump spectral bandwidth, linear and nonlinear effects can become of the same order $\left(t_{c} \sim \tau_{0}\right)$, so that the system no longer evolves in the weakly nonlinear regime and the validity of the theoretical kinetic approach becomes questionable. In the anomalous dispersion regime considered here, this may lead to the generation of a coherent soliton. This is illustrated in Fig. 8, which reports a simulation realized with the same parameters as in Fig. 7, except that the spectral bandwidth has been decreased down to $1.55 \mathrm{THz}$. We can note that the system initially tends to generate a discrete SIS, as in Fig. 7, however, at the time $T \simeq 60 T_{0}$, the first sideband of the discrete SIS suddenly leads to the generation of a coherent soliton, which is rapidly shifted toward the low frequency components. This becomes apparent in the corresponding temporal intensity profile reported in Fig. 8b, which shows that a large amplitude coherent soliton is generated. Note in particular in Fig. 8b that most of the power of the incoherent component is transferred to the coherent soliton component. This energy transfer process is strongly favoured by the Raman effect, whose underlying spectral red-shift confers a large momentum to the soliton.

\section{B. Discussion}

Let us consider the possible experimental realization of the proposed concept. While basic elements and components are readily available on the market, there are serious technical challenges and limitations for experimental implementation. Firstly, we estimate the achievable finesse in the fibre-based ring cavity. To do that we need to account for all types of losses, such as splice losses, bending losses and fibre coupler intrinsic losses. The combined splice losses together with the bending losses will amount to $\sim 0.2-0.25 \mathrm{~dB}$ per cavity round-trip. The fibre losses of $0.2 \mathrm{~dB} / \mathrm{km}$ could be neglected for 25 meter long cavity. Insertion losses on fibre coupler together with excess losses add approximately $0.25 \mathrm{~dB}$. So the best achievable fibre-based cavity finesse is around 55. Minimising the number of splices between different types of fiber and careful elimination of bending losses, one can potentially bring the finesse value up to 100. Another limiting factor is the bandwidth of the fiber-based coupler. Indeed, the typical bandwidth of a wideband fibre optical 99/1 coupler is no more than $200 \mathrm{~nm}$, which is $25 \mathrm{THz}$ or just two Raman shifts at $1550 \mathrm{~nm}$. This means that only 2 first lobes could be extracted from the cavity while further lobes will encompass much lower cavity finesse, and then the further energy transfer will be suppressed.

Limitations also arise from the available sources of stochastic pulses used to pump the cavity. One needs to have pulses of high peak power, of long duration, and of incoherent nature. The availability of such sources is really limited. Ideally the cavity should be pumped synchronously, which means that for a $25 \mathrm{~m}$ long cavity the pump pulse repetition rate is $8 \mathrm{MHz}$. For such a short cavity and low finesse the average power of the incoherent pulse should be of the order of $100 \mathrm{~W}$, which may be obtained by considering a duration of the incoherent pulse of $\sim 20-50$ ns. A possible solution is to use Nd:YAG Qswitched laser coupled into a fibre. Another possibility is a semiconductor laser, such as VECSEL, however achieving the required parameters could prove more difficult in this case. 

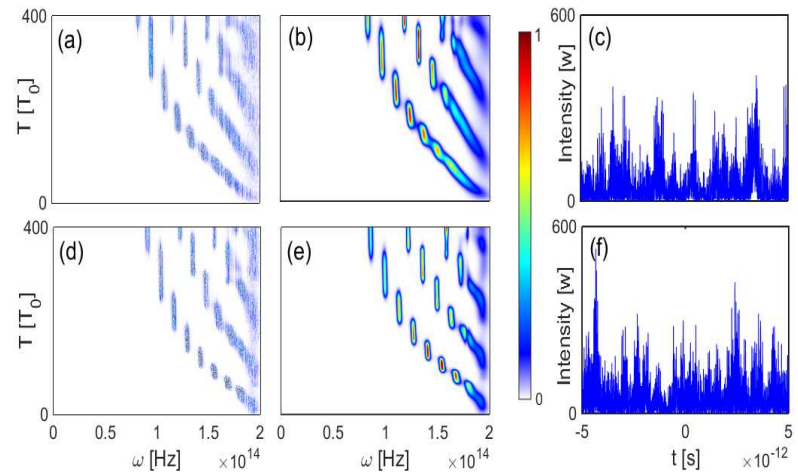

FIG. 7: Spectral dynamics obtained by simulations of NLSE (1) (including the instantaneous Kerr effect, $f_{R}=0.18$, and the Raman response function, $\eta=2.6)$ with boundary conditions (2) (1st column), corresponding MF-KE (5) (2nd column), for the optical fiber ring cavity described in the text (see Fig. 6 and Sec. VI for all parameters). The 3rd column shows the intensity temporal dynamics, $|\psi|^{2}\left(t, T=400 T_{0}\right)$, in a specific temporal window. The incoherent cavity leads to the generation of a pattern of discrete SISs with different spectral bandwidths of the incoherent pump: 1st line $6.2 \mathrm{THz} ; 2 \mathrm{nd}$ line $3.1 \mathrm{THz}$. A quantitative agreement is obtained between NLSE and MF-KE simulations without using adjustable parameters. Note in particular that no coherent solitons are generated, in contrast to Fig. 8.
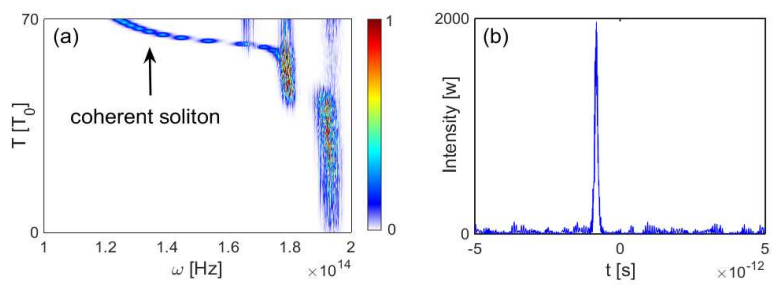

FIG. 8: Spectral dynamics obtained by simulations of NLSE (1) (including the instantaneous Kerr effect, $f_{R}=0.18$, and the Raman response function, $\eta=2.6$ ) with boundary conditions (2) (a), and corresponding intensity temporal dynamics, $|\psi|^{2}\left(t, T=70 T_{0}\right)$, for the optical fiber ring cavity described in the text (see Fig. 6 and Sec. VI for all parameters). The only difference with respect to Fig. 7 is that the spectral bandwidth of the incoherent pump has been decreased to $1.55 \mathrm{THz}$. The evolution is characterized by the generation of a coherent soliton at $T \simeq 60 T_{0}$, see the text for discussion.

\section{CONCLUSION}

We have shown that the Raman effect dominates the turbulent dynamics of an optical fiber ring cavity pumped by a strongly incoherent source. We have derived a MF$\mathrm{KE}$ that describes in detail the spectral evolution of the turbulent dynamics, which revealed a variety of behaviors, such as nonequilibrium stationary turbulent states of different nature than those predicted by the standard wave turbulence theory, the formation of spectral patterns of SISs, or the development of incoherent dispersive shock waves and incoherent collapse singularities. Note that, although dispersive shock waves are known to regularize a gradient catastrophe in a conservative physical system, here they have been shown to develop far from the stationary behavior of the cavity in the presence of forcing and damping $\left(\tau_{\text {shock }} \ll \tau_{p h}\right)$. Such a diversity of turbulent behaviors has been reported in the ideal highfinesse cavity configuration, while the formation of patterns of SISs has been found as the natural regime of operation for moderate cavity finesses. We have shown that there exist a formal analogy between the derived MF-KE accounting for forcing and damping effects inherent to the cavity configuration and the universal form of the kinetic equation describing weak Langmuir turbulence. The analysis reveals that the experimental feasibility of the demonstration of weak Langmuir optical turbulence in standard optical fiber cavities is not immediate - while basic elements and components should be readily available, the implementation of the whole experiment involves serious technical challenges. On the other hand, the exceptional high-finesse inherent to optical micro-resonators [72] would offer the possibility to explore in a relatively simple optical setting the whole richness of Langmuir turbulence [58, 61, 62]. This type of experiments should also be of interest for the important issue of the coherence properties of frequency combs in micro-resonators, whose study is the subject of a current vivid interest [77-81].
[1] B. Barviau, J. Garnier, G. Xu, B. Kibler, G. Millot, A. Picozzi, Phys. Rev. A 87, 035803 (2013).

[2] M. Erkintalo, M. Surakka, J. Turunen, A.T. Friberg, G. Genty, Opt. Lett. 37, 169 (2012).

[3] N. Akhmediev, J.M. Dudley, D.R. Solli, S.K. Turitsyn, J. Opt. 15, 060201 (2013).

[4] M. Onorato, S. Residori, U. Bortolozzo, A. Montina, F.T. Arecchi, Phys. Reports 528, 47 (2013).

[5] J.M. Dudley, F. Dias, M. Erkintalo, G. Genty, Nature Photon. 8, 755 (2014).

[6] N. Akhmediev et al., J. Opt. 18063001 (2016).
[7] J. Garnier, G. Xu, S. Trillo, A. Picozzi, Phys. Rev. Lett. 111, 113902 (2013).

[8] G. Xu, J. Garnier, S. Trillo, and A. Picozzi, Phys. Rev. A 90, 013828 (2014).

[9] G. Xu, D. Vocke, D. Faccio, J. Garnier, T. Rogers, S. Trillo, A. Picozzi, Nature Comm. 6, 8131 (2015).

[10] Y. Bromberg, Y. Lahini, E. Small, and Y. Silberberg, Nat. Photonics 4, 721 (2010).

[11] S. Derevyanko, E. Small, Phys. Rev. A 85, 053816 (2012).

[12] A. Picozzi, J. Garnier, T. Hansson, P. Suret, S. Ran- 
doux, G. Millot, D. Christodoulides, Phys. Reports 542, 1-132 (2014).

[13] A. Fratalocchi, C. Conti, G. Ruocco, S. Trillo, Phys. Rev. Lett. 101, 044101 (2008).

[14] V. Pyragaite, V. Smilgevicius, R. Butkus, A. Stabinis, A. Piskarskas, Phys. Rev. A 88, 023820 (2013).

[15] T. Hansson, M. Lisak, D. Anderson, Phys. Rev. Lett. 108, 063901 (2012).

[16] J. Laurie, U. Bortolozzo, S. Nazarenko, S. Residori, Phys. Rep. 514, 121 (2012).

[17] Y. Silberberg, Y. Lahini, Y. Bromberg, E. Small, R. Morandotti, Phys. Rev. Lett. 102, 233904 (2009).

[18] P. Aschieri, J. Garnier, C. Michel, V. Doya, A. Picozzi, Phys. Rev. A 83, 033838 (2011).

[19] L.G. Wright, D.N. Christodoulides, F.W. Wise, Nature Photon. 9, 306 (2015).

[20] P. Suret, A. Picozzi, S. Randoux, Opt. Express 1917852 (2011).

[21] S. Randoux, P. Walczak, M. Onorato, P. Suret, Phys. Rev. Lett. 113, 113902 (2014).

[22] P. Walczak, S. Randoux, P. Suret, Phys. Rev. Lett. 114, 143903 (2015).

[23] C. Conti, M. Leonetti, A. Fratalocchi, L. Angelani, G. Ruocco, Phys. Rev. Lett. 101, 143901 (2008).

[24] S. Babin, D. Churkin, A. Ismagulov, S. Kablukov, E. Podivilov, J. Opt. Soc. Am. B 24, 1729 (2007).

[25] S. Wabnitz, Opt. Lett, 39, 1362-1365, (2014).

[26] L. Gil, G.L. Lippi, Phys. Rev. Lett. 113, 213902 (2014).

[27] C.J. Gibson, A.M. Yao, G.L. Oppo, Phys. Rev. Lett. 116, 043903 (2016).

[28] S. A. Babin, D. V. Churkin, A. E. Ismagulov, S. I. Kablukov and E. V. Podivilov, Opt. Lett, 31, 30073009, (2006).

[29] D.V. Churkin, I.V. Kolokolov, E.V. Podivilov, I.D. Vatnik, S.S. Vergeles, I.S. Terekhov, V.V. Lebedev, G. Falkovich, M.A. Nikulin, S.A. Babin, S.K. Turitsyn, Nature Comm. 6, 6214 (2015).

[30] E. Turitsyna, S. Smirnov, S. Sugavanam, N. Tarasov, X. Shu, S. Babin, E. Podivilov, D. Churkin, G. Falkovich, S. Turitsyn, Nat. Photon. 7, 783 (2013).

[31] S.V. Smirnov, N. Tarasov, D.V. Churkin, Opt. Express 23, 27606 (2015).

[32] R. Weill, B. Fischer, O. Gat, Phys. Rev. Lett. 104, 173901 (2010).

[33] G. Oren, A. Bekker, B. Fischer, Optica 1, 145 (2014).

[34] A. Schwache, F. Mitschke, Phys. Rev. E 55, 7720 (1997).

[35] S. Kobtsev, S. Kukarin, S. Smirnov, S. Turitsyn, A. Latkin, Opt. Exp. 17, 20707 (2009).

[36] P. Grelu, N. Akhmedeiv, Nature Photon. 6, 84 (2012).

[37] A. Aragoneses, L. Carpi, N. Tarasov, D.V. Churkin, M.C. Torrent, C. Masoller, and S.K. Turitsyn, Phys. Rev. Lett. 116, 033902 (2016).

[38] J. Klaers, J. Schmitt, F. Vewinger, and M. Weitz, Nature 468, 545 (2010).

[39] I. Carusotto, C. Ciuti, Rev. Mod. Phys. 85, 299 (2013).

[40] V.E. Zakharov, V.S. L'vov and G. Falkovich, Kolmogorov Spectra of Turbulence I (Springer, Berlin, 1992).

[41] S. Nazarenko, Wave Turbulence (Springer, Lectures Notes in Physics, 2011).

[42] C. Michel, M. Haelterman, P. Suret, S. Randoux, R. Kaiser, and A. Picozzi, Phys. Rev. A 84, 033848 (2011).

[43] A. Picozzi, J. Garnier, Phys. Rev. Lett. 107, 233901
(2011).

[44] D.B. Soh, J.P. Koplow, S.W. Moore, K.L. Schroder, W.L. Hsu, Opt. Exp. 18, 22393 (2010).

[45] C. Michel, P. Suret, S. Randoux, H.R. Jauslin, A. Picozzi, Opt. Lett. 352367 (2010).

[46] A. Picozzi, S. Pitois, G. Millot, Phys. Rev. Lett. 101, 093901 (2008).

[47] J. Garnier, A. Picozzi, Phys. Rev. A 81, 033831 (2010).

[48] C. Michel, B. Kibler, A. Picozzi, Phys. Rev. A 83, 023806 (2011).

[49] B. Kibler, C. Michel, A. Kudlinski, B. Barviau, G. Millot, A. Picozzi, Phys. Rev. E 84, 066605 (2011).

[50] G. Xu, J. Garnier, M. Conforti, A. Picozzi, Opt. Lett. 39, 4192 (2014).

[51] G.P. Agrawal, Nonlinear Fiber Optics, 5th ed. (Academic Press, 2012).

[52] T. Carmon, M. Soljacic, M. Segev, Phys. Rev. Lett. 89, 183902 (2002).

[53] M. Conforti, A. Mussot, J. Fatome, A. Picozzi, S. Pitois, C. Finot, M. Haelterman, B. Kibler, C. Michel, and G. Millot, Phys. Rev. A 91, 023823 (2015).

[54] L.A. Lugiato, R. Lefever, Phys. Rev. Lett. 58, 2209 (1987).

[55] M. Haelterman, S. Trillo, and S. Wabnitz, Opt. Commun. 91, 401 (1992).

[56] V. E. Zakharov, Zh. Eksp. Teor. Fiz. 62, 1745 (1972) [Sov. Phys. JETP 35, 908 (1972)].

[57] V.E. Zakharov, S.L. Musher, A.M. Rubenchik, Phys. Reports 129, 285 (1985).

[58] S.L. Musher, A.M. Rubenchik, and V.E. Zakharov, Phys. Reports 252, 177 (1995).

[59] C. Montes, Astrophys. J 216, 329 (1977).

[60] C. Montes, Phys. Rev. A 20, 1081 (1979).

[61] M.V. Goldman, Rev. Mod. Phys. 56, 709 (1984).

[62] P. A. Robinson, Rev. Mod. Phys. 69, 507 (1997).

[63] I. Langmuir, Science 87, 119-123 (1938).

[64] A.D.D. Craik, S. Leibovich, J. Fluid Mech. 73, 401-426 (1976).

[65] J.C. Mc Williams, P.P. Sullivan, C.-H. Moeng, J. Fluid Mech. 334, 1-30 (1997).

[66] A. Y. Wong, P. Y. Cheung, Phys. Rev. Lett. 52, 1222 (1984).

[67] L. N. Vyacheslavov, V. S. Burmasov, I. V. Kandaurov, E. P. Kruglyakov, O. I. Meshkov, S. S. Popov, A. L. Sanin, Plasma Phys. Controlled Fusion 44, B279 (2002).

[68] M. P. Sulzer and J. A. Fejer, J. Geophys. Res. 99, 15035 (1994).

[69] B. Isham, C. La Hoz, M. T. Rietveld, T. Hagfors, and T. B. Leyser, Phys. Rev. Lett. 83, 2576 (1999).

[70] M. T. Rietveld, B. Isham, H. Kohl, C. La Hoz, and T. Hagfors, J. Geophys. Res. 105, 7429 (2000).

[71] B. Isham, M. T. Rietveld, P. Guio, F. R. E. Forme, T. Grydeland, E. Mjolhus, Phys. Rev. Lett. 108, 105003 (2012).

[72] T. Herr, V. Brasch, J.D. Jost, C.Y. Wang, N.M. Kondratiev, M.L. Gorodetsky, T.J. Kippenberg Nature Photon. 8, 145 (2014).

[73] C. Bao et al., Opt. Lett. 39, 6126 (2014).

[74] T. Hansson, D. Modotto, S. Wabnitz, Opt. Lett. 39, 6747 (2014).

[75] Y.K. Chembo, C.R. Menyuk, Phys. Rev. A 87, 053852 (2013).

[76] C. Milian, A. V. Gorbach, M. Taki, A. V. Yulin, D. V. Skryabin, Phys. Rev. A 92, 033851 (2015). 
[77] A. B. Matsko, W. Liang, A. A. Savchenkov, and L. Maleki, Opt. Lett. 38, 525 (2013).

[78] T. Q. Banh, K. Suzuki, T. Shioda, Opt. Comm. 296, 1 (2013).

[79] M. Erkintalo, S. Coen, Opt. Lett. 39, 283 (2014).

[80] W. Loh, P. Del'Haye, S. B. Papp, S. A. Diddams Phys. Rev. A 89, 053810 (2014).

[81] D. Burghoff et al., Nature Photon. 8, 462-467, (2014).

[82] K. J. Blow, D. Wood, IEEE J. Quantum Electron. 25, 2665-2673 (1989).

[83] T. Arecchi, S. Boccaletti, and P. Ramazza, Phys. Rep. 318, 1 (1999).

[84] F. Leo, S. Coen, P. Kockaert, S.P. Gorza, P. Emplit, M. Haelterman, Nature Photon. 4, 471 (2010).

[85] F. Leo, A. Mussot, P. Kockaert, P. Emplit, M. Haelterman, M. Taki, Phys. Rev. Lett. 110, 104103 (2013).

[86] J.K. Jang, M. Erkintalo, S.G. Murdoch, S. Coen, Nature Photonics 7, 657-663 (2013).

[87] Z. Liu, F. Leo, S. Coulibaly, M. Taki Phys. Rev. A 90, 033837 (2014).

[88] F. Leo, T. Hansson, I. Ricciardi, M. De Rosa, S. Coen, S. Wabnitz, and M. Erkintalo Phys. Rev. Lett. 116, 033901 (2016).

[89] K. Hammani, A. Picozzi, C. Finot, Opt. Commun. 284, 2594 (2011)

[90] B. Rumpf, A.C. Newell, Phys. Rev. Lett. 87, 054102 (2001).

[91] K. Hammani, B. Kibler, C. Finot, A. Picozzi, Phys. Lett. A 374, 3585 (2010).

[92] B. Kibler, K. Hammani, C. Michel, C. Finot, A. Picozzi, Phys. Lett. A 375, 3149 (2011).

[93] B. Barviau, B. Kibler, A. Picozzi, Phys. Rev. A 79, 063840 (2009).

[94] A.K. Abeeluck and C. Headley, Opt. Lett. 30, 61 (2005).

[95] A. Aalto, G. Genty, J. Toivonen, Opt. Express 18, 1234 (2010).

[96] E.M. Dianov and M.Prokhorov, IEEE J. Sel. Quantum. Electron. 6, 1022 (2000).

[97] A.F.J. Runge, C. Aguergaray, N.G.R. Broder- ick, M. Erkintalo, Opt. Lett. 39, 319 (2014).

[98] S.M. Spillane et al., Nature 415, 621 (2002).

[99] B. Min, T.J. Kippenberg, K.J. Vahala, Opt. Lett. 28, 1507 (2003).

[100] R. J. Taylor, D.R. Baker, and H. Ikezi, Phys. Rev. Lett. 24, 206 (1970).

[101] J. L. Hammack, H. Segur, J. Fluid Mech. 65, 289 (1974).

[102] J.E. Rothenberg, D. Grischkowsky, Phys. Rev. Lett. 62, 531 (1989).

[103] W. Wan, S. Jia, J.W. Fleischer, Nat. Phys. 3, 46 (2007).

[104] N. Ghofraniha, C. Conti, G. Ruocco, and S. Trillo, Phys. Rev. Lett. 99, 043903 (2007).

[105] G.A. El, A. Gammal, E.G. Khamis, R.A. Kraenkel, A.M. Kamchatnov, Phys. Rev. A 76, 053813 (2007).

[106] C. Conti, A. Fratalocchi, M. Peccianti, G. Ruocco, and S. Trillo, Phys. Rev. Lett. 102, 083902 (2009).

[107] P. Whalen, J.V. Moloney, A.C. Newell, K. Newell, M. Kolesik, Phys. Rev. A 86, 033806 (2012).

[108] N. Ghofraniha, S. Gentilini, V. Folli, E. Del Re, C. Conti, Phys. Rev. Lett. 109, 243902 (2012).

[109] S. Wabnitz, C. Finot, J. Fatome, G. Millot, Phys. Lett. A 377, 932-939 (2013).

[110] M. Conforti, F. Baronio, S. Trillo, Phys. Rev. A 89, 013807 (2014).

[111] J. Fatome, C. Finot, G. Millot, A. Armaroli, S. Trillo, Phys. Rev. X 4, 021022 (2014).

[112] M. Conforti, A. Mussot, A. Kudlinski, S. Trillo, Sci. Rep. 5, 9433 (2015).

[113] S. Malaguti, G. Bellanca, S. Trillo, Opt. Lett. 39, 2475 (2014).

[114] S. Malaguti, M. Conforti, S. Trillo, Opt. Lett. 39, 5626 (2014).

[115] V.E. Lobanov, G. Lihachev, T. J. Kippenberg, M.L. Gorodetsky, Opt. Exp. 23, 7713 (2015).

[116] Y. Liu et al., Optica 1, 137 (2014).

[117] X. Xue et al., Nature Phot. 9, 594 (2015).

[118] P. Constantin, P. Lax, A. Majda, Commun. Pure Appl. Math. 38, 715 (1985). 